\title{
Bloqueos de extremidad superior
}

\author{
Julián Aliste MD. ${ }^{1}$, Daniela Bravo MD. ${ }^{1}$, Sebastián Layera MD. ${ }^{1}$
}

Palabras clave: Bloqueos de plexo braquial, bloqueos de nervio periférico, extremidad superior, anestesia regional.

Términos Mesh: brachial plexus blocks.

\section{Introducción}

os bloqueos de nervios periféricos son altamente populares en cirugía de extremidad superior (ES) dado que, si son bien ejecutados, más allá de sólo otorgar analgesia postoperatoria pueden producir anestesia quirúrgica, la que se puede complementar con grados variables de sedación y evitar así la anestesia general y sus efectos adversos.

Estos bloqueos son ideales en casos de cirugía ambulatoria, ya que proveen altas más precoces, mejor control del dolor, y menos efectos adversos[1]. El uso de coadyuvantes puede prolongar su efecto hasta 24 horas[1]. Sin embargo, si es requerido un efecto más duradero, se debe proceder con un bloqueo continuo mediante la infusión de anestésicos locales (ALs) a través de un catéter perineural[1].

Un adecuado conocimiento de la anatomía del plexo braquial (PB) y de la inervación osteomuscular permite planificar de manera óptima el bloqueo acorde a la cirugía y a las características del paciente.

Los abordajes más frecuentemente utilizados para el PB son interescalénico, supraclavicular, infraclavicular y axilar[1].

Respecto a la técnica de neurolocalización, en bloqueos de PB el ultrasonido (US) ha demostrado acortar los tiempos de ejecución del bloqueo, reducir los pases de aguja, menor dolor asociado al procedimiento, menor incidencia de punción vascular, bloqueo sensitivo más rápido y mayor tasa de éxito[2].
Además, la evidencia favorecería aquellas técnicas con menos inyecciones[2].

\section{Anatomía}

En el $75 \%$ de los individuos el plexo braquial (PB) (Figura 1) deriva de los ramos anteriores de las raíces desde C5 a T1 con contribuciones variables de C4 y T2[3]. La distancia que recorren las raíces (ramos anteriores) desde el foramen hasta conformar los troncos del plexo es variable, siendo habitualmente entre 30 $\mathrm{mm}$ y $60 \mathrm{~mm}[3]$.

La duramadre del manguito dural de cada raíz se transforma en el epineuro y perineuro en su trayecto hacia la periferia[3].

Antes de formarse los troncos, desde las raíces emergen el nervio torácico largo (NTL) (C5-C6-C7) que inerva el músculo serrato anterior y el nervio dorsoescapular (NDE) (C5) que inerva los músculos romboides y elevador escapular. Ambos nervios tienen un trayecto a través del músculo escaleno medio (MEM) o entre este último y el escaleno posterior, lo que debe considerarse en bloqueos a este nivel. Además, hasta en un $20 \%$ de los casos el nervio frénico (NF) puede provenir desde raíces del PB y no del cervical. Por último, las raíces C5-C6-C7-C8 inervan los músculos escalenos y longus colli[3].

En su trayecto entre el MEM y el músculo escaleno anterior (MEA) las raíces pasan a conformar los

1 Departamento de Anestesiología y Medicina Perioperatoria, Universidad de Chile.

Fecha de ingreso: 08 de noviembre de 2019

Fecha de aceptación: 10 de noviembre de 2019

\section{ORCID}

https://orcid.org/0000-0001-6355-1270

Correspondencia:

Julián Aliste MD

Email: julian.aliste@uchile.com 


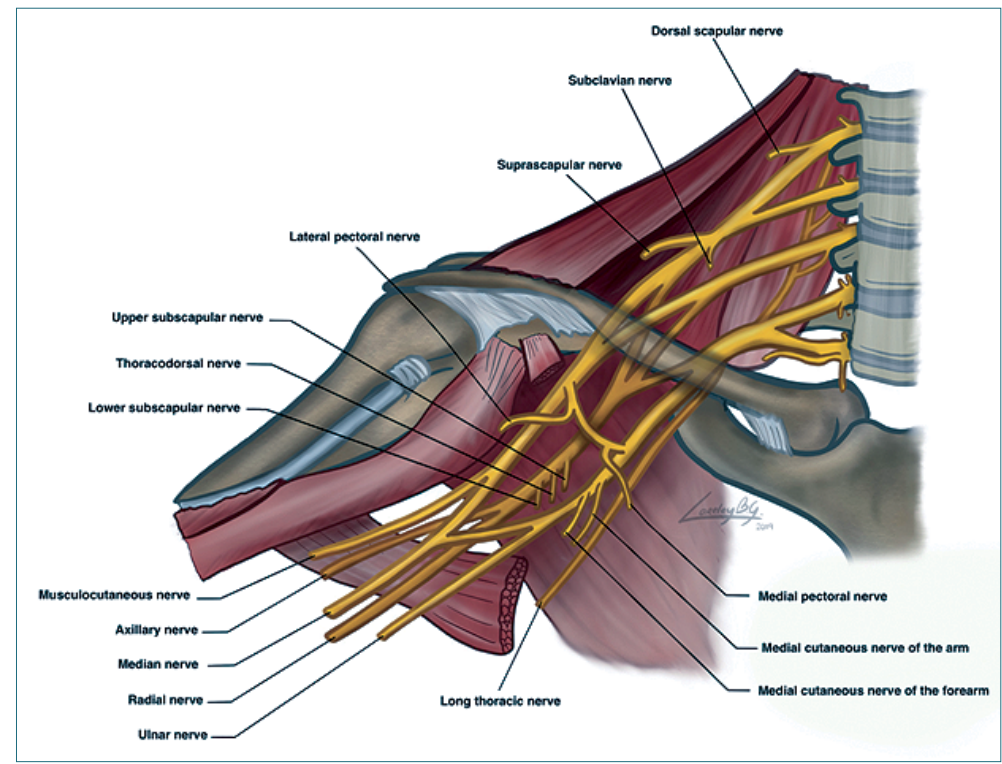

Figura 1. Plexo braquial.

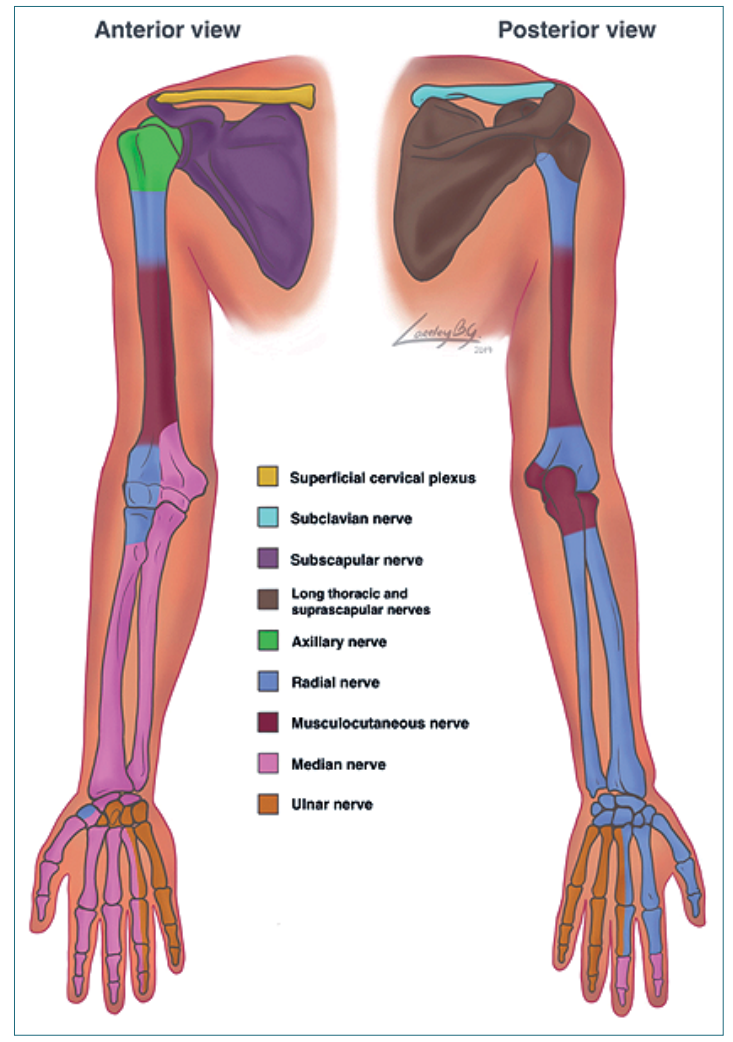

Figura 2. Inervación ósea extremidad superior. Visión anterior y posterior. troncos del PB. A este nivel desde el tronco superior emergen el nervio supraescapular (NSE) que inerva los dos tercios posteriores de la articulación del hombro, la articulación acromio-clavicular y los músculos supra e infraespinoso, y el nervio subclavio que inerva el músculo del mismo nombre y la cara póstero-inferior de la clavícula[3],[4].

Aún a nivel interescalénico, cada tronco origina una división anterior (flexores) y posterior (extensores) las cuales son fácilmente identificables en su paso por sobre la primera costilla, lateral a la arteria subclavia, organizadas en forma de racimo. A nivel de divisiones no se originan nervios terminales en general.

En su paso retroclavicular, las divisiones se unirán para formar los cordones o fascículos; medial (C5-C6C7), lateral (C5-C6-C7-C8-T1) y posterior (C8-T1), que inicialmente a nivel costoclavicular mantienen una relación compacta y aún lateral a la arteria subclavia (ASC), para luego distribuirse rodeando a esta última ya como arteria axilar (AA)[5].

A pesar de sus nombres, los cordones a nivel infraclavicular no necesariamente se ubican en esa relación con la $A A$, encontrándose más frecuentemente en una ubicación posterolateral, pero en un mismo plano fascial con la arteria[6].

El cordón lateral $(\mathrm{CL})$ originará el nervio pectoral lateral (NPL), que inerva el músculo pectoral mayor y emite un ramo articular para la articulación del hombro. Además de este cordón emerge el nervio musculocutáneo (NMC) que a nivel axilar abandona la vaina periarterial axilar y penetra en los músculos flexores 
del antebrazo, para luego inervar la cara distal anterior del húmero, la articulación del codo por anterior y, por último, la región cutánea lateral del antebrazo. Además, el CL origina la porción lateral del nervio mediano (NM)[3].

El cordón medial (CM) emite varios ramos terminales. Entre ellos el nervio pectoral medial que inerva principalmente el músculo pectoral menor. Además, origina los nervios cutáneos mediales del brazo y antebrazo, y la porción medial del NM.

El cordón posterior (CP) origina los nervios subescapulares superior e inferior, que inervan la cara anterior de la escápula, los músculos en esta región y parte de la cápsula articular del hombro. Además, se originan el nervio tóracodorsal (NTD), que inerva el músculo dorsal ancho, y el nervio axilar (NA), que inerva la cabeza y cuello humeral, el músculo deltoides y la cápsula glenohumeral[3].

Finalmente, a nivel axilar, desde el borde lateral del músculo pectoral mayor, encontramos sólo los nervios que persisten rodeando la arteria axilar. El nervio radial (NR) que inerva la mayor parte del dorso óseo, cutáneo y músculos extensores del antebrazo, muñeca y región dorso-lateral de la mano. El NM que inerva parte del codo, la cara anterior ósea y muscular del antebrazo y la región ventro-lateral de la mano. El nervio ulnar (NU) inerva los músculos flexores mediales de la muñeca y mano junto con el aductor del pulgar y los encargados de la ulnarización del carpo más el componente óseo, músculo-tendinoso y cutáneo de la región medial de la mano[3].

\section{Cintura escapular}

La inervación de las estructuras que componen la cintura escapular proviene principalmente del PB, pero también puede recibir aportes desde el plexo cervical superficial y el ramo anterior de la segunda raíz torácica (T2).

El plexo cervical superficial, a través de los nervios supraclaviculares, inerva la cara anterior de clavícula y la piel de la capa del hombro[3].

La raíz T2 puede inervar la articulación esternoclavicular y además conforma el nervio intercostobraquial que inerva la axila y la región cutánea medial del brazo[3].

A su vez el PB es el principal encargado de la inervación de la articulación del hombro a través de los nervios supraescapular, axilar, pectoral lateral y subescapulares[7]. Además, el nervio subclavio inerva la cara posterior de la clavícula[3],[4]. Dado que los nervios supraescapular y subclavio emergen precoz- mente a nivel del tronco superior, los bloqueos por sobre la clavícula (interescalénico y supraclavicular) son altamente eficaces en otorgar analgesia en cirugías de hombro y clavícula. Sin embargo, bloqueos más específicos y a la vez más distales que intentan evitar la parálisis hemidiafragmática (PHD) secundaria al bloqueo del NF han demostrado ser alternativas analgésicas válidas para esta región, aunque no todas equivalentes al bloqueo interescalénico[8].

\section{Territorio medio-humeral a distal}

Para el bloqueo de esta región debe considerarse un adecuado bloqueo de los nervios más terminales del PB, los nervios radial, mediano, musculocutáneo y ulnar.

En general, si se desea anestesia quirúrgica para una cirugía con isquemia es esencial conocer los territorios inervados por estos cuatro nervios, las características del procedimiento quirúrgico y el nivel en que se instalará el torniquete. Además, si el abordaje quirúrgico se ejecuta por la cara medial del brazo o antebrazo, en pacientes despiertos o levemente sedados es crucial el bloqueo de los nervios cutáneos mediales que también provienen del PB.

Respecto al dolor producido por el mango de isquemia, cuando se instala el torniquete en el brazo es necesario el bloqueo del NMC y del NR. En cambio, si el torniquete se ubica a nivel del antebrazo se requiere que el NR y el NM estén bloqueados.

Cuando la cirugía se realiza bajo anestesia general, tanto para bloqueos prequirúrgicos como postquirúrgicos, se debe considerar que el bloqueo del NMC es necesario en cirugía a nivel del húmero terminal y codo, pero no en cirugía distal a este último y así se puede preservar la flexión del codo en el postoperatorio.

En cirugía de mano, el bloqueo del NU es necesario para procedimientos en cuarta o quinta metacarpo-falanges, pero no así para tres primeras.

\section{Pared torácica}

Algunos nervios del plexo braquial inervan músculos de la pared torácica y han sido considerados relevantes en algunas cirugías a este nivel. Entre estos nervios se incluyen los nervios pectorales lateral y medial que inervan los músculos pectorales y que su bloqueo podría cumplir cierto rol analgésico, aún en discusión, en procedimientos que comprometan tanto el músculo o su fascia ya sea por daño directo 
o distensión. En esta misma línea, cirugías reconstructivas de mama que utilicen injertos de músculo dorsal ancho podrían favorecerse del bloqueo del nervio tóracodorsal.

Sin embargo, en general estos nervios se bloquean en su trayecto a nivel del tórax y se les considera bloqueos de tronco y no se discutirán en esta revisión.

\section{Bloqueo interescalénico}

El bloqueo interescalénico (BIE) del plexo braquial representa el estándar en cirugía de hombro[7],[8] en términos de analgesia, satisfacción y menor estadía hospitalaria. Dado su elevada incidencia de efectos colaterales, su indicación debe ser siempre sopesada según los antecedentes de cada paciente.

Profundo a la fascia prevertebral, entre el MEA y el MEM se ubica la imagen ecográfica clásica (estructuras redondeadas hipoecoicas) del PB que pueden representar tanto las raíces en su transición a troncos o a estos últimos previo a dividirse. La hipoecogenicidad de éstos, se explicaría por una mayor concentración fascicular que de tejido conectivo, lo cual determinaría su mayor susceptibilidad a daño traumático con secuelas. A este nivel el plexo está dentro de una vaina de tejido conectivo. El inyectar dentro de esta vaina fascial produciría una mayor duración de bloqueo[9] y mayor posibilidad de distribución cefálica del AL[7]. Cuando se observan tres estructuras hipoecoicas (tipo semáforo), estas pueden corresponder a C5, C6, C7 o C5 y una raíz C6 subdividida. La raíz de C6 tiende a ser más grande que C5. Esto último puede explicar que inyecciones entre los puntos hipoecoicos medio e inferior del "semáforo", dado que estarían rodeados por el epineuro/duramadre de C6, podrían extenderse hacia el neuroaxis y explicar bloqueos bilaterales reportados en la literatura[10].

A la altura clásica de inyección (nivel del cricoi- des) el NF emerge del surco interescalénico, anterior al plexo braquial a una distancia promedio de dos milímetros[7]. En su recorrido hacia distal, el nervio se aleja del plexo a razón de tres milímetros por cada centímetro de recorrido sobre el MEA[7] y bajo la fascia prevertebral, para luego continuar su recorrido intratorácico. Esta proximidad explicaría el frecuente bloqueo frénico con abordajes del plexo por sobre la clavícula alcanzando hasta un $100 \%$ en BIE[7],[8].

Por último, es importante recordar que hasta en un $90 \%$ de los pacientes, tanto en la superficie del MEM como en su interior se puede visualizar estructuras nerviosas que con mayor frecuencia corresponde al NDE y en menor medida con el NTL[11], por lo tanto, en todos los abordajes a través del MEM deben evitarse estructuras que pudieran corresponder a estos nervios. Ante la duda el uso de neuroestimulación, puede ayudar a diferenciarlos[11].

La guía por US en BIE ha demostrado ser superior a neuroestimulación (NE), permitiendo menores tiempos de ejecución, menos pases de aguja, menos punciones vasculares, menos dolor asociado al procedimiento, latencia más corta, la posibilidad de disminuir hasta cinco veces el volumen efectivo mínimo (VEM) de ALs, y menos catéteres disfuncionantes[2].

Con el paciente mirando contralateralmente, el transductor se ubica a nivel del cartílago cricoides y se escanea lateralmente (Figura 3A) hasta identificar el surco interescalénico y el plexo a este nivel (Figura 3B).

Se han descrito abordajes posteriores, tipo paravertebral, donde el objetivo de punción ecográfica es finalmente el mismo, raíces C5-C6 y cuya ventaja radicaría en generar mayor trayecto en la instalación de catéteres manteniendo el sitio de entrada más alejado del campo quirúrgico[12]. También está descrito el abordaje fuera de plano en el cual obteniendo la imagen de raíces/troncos, se realiza una punción de medial a lateral fuera de plano, la cual también sería ventajosa para la instalación de catéteres[13]. Por úl-

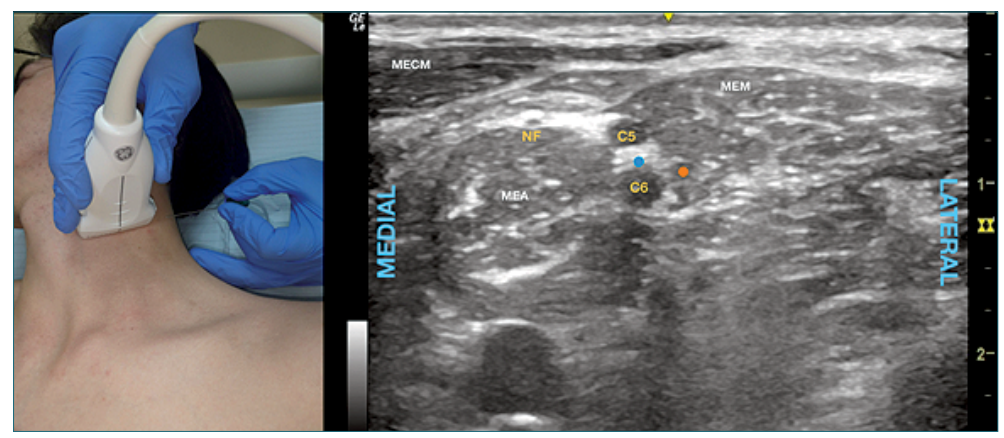

Figura 3. A. Posición transductor BIE; B. Sonoanatomía BIE: MEA: músculo escaleno anterior; MEM: músculo escaleno medio; MECM: músculo esternocleidomastoideo; C5: $5^{\text {a }}$ raíz cervical; C6: $6^{\text {a }}$ raíz cervical; NF: nervio frénico; Punto azul: sitio de inyección intrafascial; Punto naranjo: punto de inyección extrafascial. 
timo, lo más frecuente es el abordaje en plano desde lateral a medial con la misma imagen de raíces/tron$\cos [1]$.

Estudios de dosis han determinado que es posible generar un bloqueo sensitivo y motor preoperatorio de las raíces de C5 y C 6 con volúmenes tan bajos como 0,95 $\mathrm{mL}$ de bupivacaina 0,5\% distribuido en 2 inyecciones (entre C5-6 y C6-7). Sin embargo, el mismo estudio determinó que al menos $3 \mathrm{~mL}$ son necesarios para una adecuada analgesia a las 6 horas postoperatorias, pero con un intervalo de confianza amplio $(0,5$ a $11,5 \mathrm{~mL})$ y que hasta $4 \mathrm{~mL}$ podría correlacionarse con ausencia de bloqueo frénico[14]. Un estudio similar concluyó que $5 \mathrm{ml}$ de ropivacaina $0,75 \%$ distribuido en tres inyecciones guiadas a cada tronco poseía una eficacia similar con respecto a anestesia quirúrgica con una duración media de la analgesia postoperatoria de 10 horas siendo esta última invariable respecto al volumen[15]. Sin embargo, otros estudios que han evaluado la relación volumen/ masa y duración de bloqueo han determinado que, aunque esta relación existe no es lineal y por ejemplo, cuadriplicar la dosis de ropivacaina 0,375\% (de 10 a $40 \mathrm{~mL}$ ) sólo prolongó en un $50 \%$ el efecto, de 10 a $15 \mathrm{~h}$. Un resultado similar se logra duplicando la concentración, pero manteniendo el volumen[16].

Dado que volúmenes menores de $10 \mathrm{~mL}$ a pesar de generar bloqueos quirúrgicos exitosos se pueden asociar a mayor incidencia de dolor postoperatorio en bloqueos de dosis única[16] y que otros estudios no han demostrado que disminuyendo el volumen se logre disminuir la incidencia de parálisis hemidiafragmática lo suficiente[7], pareciera más recomendable no transar en el volumen inyectado si una técnica continua no será utilizada para mantener la calidad analgésica en el tiempo. Por otro lado, si el riesgo de parálisis frénica es prohibitivo en un determinado paciente, es razonable mejor recurrir a alguna técnica de bloqueo o analgesia alternativa al BIE.

Inyecciones intrafasciales prolongarían el efecto de bloqueos con dosis única[9]. Sin embargo, bloqueos únicos, extra-paraneurales, inyectando posterior al plexo braquial disminuyen la incidencia de parálisis frénica[17]. Respecto al número de punciones se ha descrito que tanto una, dos y tres inyecciones, ya sea entre $C 5$ y $C 6$, entre $C 5-C 6$ y C6-C7 o dirigidas a cada tronco respectivamente poseen una adecuada eficacia, sin embargo, no existen estudios comparativos entre ellas. Dada la posibilidad de que lo interpretado como C6-C7 o tronco medio-tronco inferior corresponda a una subdivisión de C6 compartiendo un epineuro común[10], recomendamos una sola inyección entre C5-C6 por ser eficaz y seguro.
Dado que la severidad del dolor postoperatorio en cirugías de hombro abiertas o artroscópicas tiende a durar más de 24 horas BIE es uno de los bloqueos de PB con más evidencia apoyando el uso de catéteres e infusión continua de ALs. Hasta ahora no existe suficiente literatura comparando bloqueos continuos contra bloqueos simples con coadyuvancia.

La forma más frecuente de instalación de catéteres guiados por US es desde postero-lateral hacia antero-medial, pudiendo dejarse el catéter dentro o fuera de la vaina que rodea el plexo. Esto último disminuye la incidencia de PHD, pero no la previene completamente[18].

Las concentraciones y volúmenes de infusión empleados son altamente variables, pero conceptualmente si un catéter está bien ubicado y no se desplaza, no se requerirían concentraciones ni volúmenes elevados para obtener una adecuada analgesia.

Debido a la alta movilidad del cuello y la poca profundidad del plexo en la mayoría de los pacientes, la frecuencia de desplazamiento con retiro accidental de catéteres a este nivel es alta. Incluso, catéteres instalados preoperatorios pueden salirse durante el intraoperatorio o con el retiro de los campos estériles dada la cercanía con el sitio quirúrgico.

Dentro de los efectos colaterales, ninguna técnica ha logrado disminuir a menos de $20 \%$ la PHD con BIE en única[8] o 15\% con BIEC[18], siendo habitual un $90-100 \%$ una técnica estándar. A esto se asocia frecuentemente (también dependiente de volumen y sitio de administración) la presencia de disfonía por parálisis del nervio laríngeo recurrente ipsilateral y síndrome de Horner por bloqueo de la cadena simpática cervical y sus ganglios.

Respecto a las complicaciones traumáticas por la punción, el correcto uso del US, ejecutando una adecuada sonoidentificación de estructuras vasculares y neurológicas permitiría disminuirlas. Es recomendable evitar estructuras que pudieran ser nervios en relación con el MEM en el trayecto hacia el plexo[11], evitar que la aguja avance hasta el MEA donde podría dañarse el NF[19], no inyectar entre una segunda y tercera estructura hipoecoica dentro de la vaina del plexo[10] y esforzarse por identificar estructuras vasculares que no siempre son muy evidentes en esta región.

\section{Bloqueo supraclavicular}

El bloqueo supraclavicular (BSC) del plexo braquial guiado por US es altamente popular dada su eficacia, eficiencia y replicabilidad tanto para anestesia o anal- 
gesia distal al hombro. Además, evidencia reciente lo ha ratificado como equivalente a BIE en analgesia postoperatoria en cirugía artroscópica de hombro[8].

El BSC aborda el plexo cuando los troncos se dividen, aun estando entre los músculos escalenos, pero más cercano al nivel de la inserción en la primera costilla donde, además, encontramos la ASC que consistentemente toma una posición medial al $\mathrm{PB}$, quedando este último además muy próximo a la pleura. A esta altura el NF se encuentra en al borde antero-medial del escaleno anterior y el NSE tiende a encontrarse en relación con el músculo omohioideo en su trayecto hacia la escotadura escapular[20].

A pesar de ser posible ejecutar un BSC con guía exclusiva de NE logrando una adecuada eficacia[3], la cercanía de la ASC y la pleura aumenta el riesgo de complicaciones serias como hematoma y neumotórax con técnicas ciegas. Así, la adición de US aparte de disminuir los tiempos de procedimiento permite aumentar la seguridad[2].

El transductor se ubica en la fosa supraclavicular, inicialmente paralelo a la clavícula en su tercio medial con un grado variable de inclinación caudal (buscando la primera costilla) (Figura 4A) para luego, dependiendo en qué punto de la curvatura costal se encuentre la ASC y el PB, pudiendo ser necesario rotar el transductor para optimizar la imagen e idealmente identificar la arteria y plexo apoyados en una línea eco refringente con sombra acústica (costilla) lo más extensa posible y no sobre líneas ecorrefringentes sin sombra (pleura), lo que aumenta el margen de seguridad de la punción (Figura 4B).

Se han descrito y comparado tanto el abordaje medial como lateral (o posterior dependiendo del grado de rotación de transductor) con similar eficacia[2]. No obstante, lo más habitual es el abordaje en plano desde lateral a medial.

Estudios con metodología validadas de búsqueda de dosis han determinado que $27 \mathrm{~mL}$ de bupivacaína 0,5\%[21] o $32 \mathrm{~mL}$ de lidocaína 1,5\%[22] representan dosis efectivas o VEM 95\% y 90\% respectivamente, en términos de volumen para anestesia quirúrgica.

Cuando el bloqueo tiene un objetivo analgésico o complementario a anestesia general, pueden ser utilizados volúmenes o concentraciones menores.

EI BSC es altamente eficaz, independiente del sitio y número de inyecciones, al utilizar una dosis adecuada de AL. Sin embargo, si se desea disminuir al máximo la latencia, múltiples inyecciones dentro del racimo de divisiones es lo más recomendado[23]. No obstante, si se dispone de tiempo y se prefiere evitar múltiples inyecciones muy cerca de fascículos, una inyección en el ángulo entre costilla y arteria subclavia (corner pocket) sería también altamente eficaz[24]. En el caso de querer emplear un BSC para analgesia de hombro se ha determinado que inyectando $20 \mathrm{~mL}$ de $A L$ distribuidos posterolateralmente al PB evitando difusión hacia anterior es equivalente a BIE, pero con 0\%-9\% de PHD[7],[25].

A diferencia del BIE, en el BSC el PB está más disperso lo cual, al igual que para las dosis únicas, en bloqueos continuos se pudiera requerir mayor volumen para rodear adecuadamente todos sus componentes, lo cual debe considerarse en el esquema de infusión a utilizar.

Cuando se requiere un bloqueo continuo de ES distal al hombro, este abordaje permite generar adecuados trayectos con el catéter y menor riesgo de desplazamiento y disfunción en comparación con BIE, sin embargo, en cirugía de hombro dado su cercanía con el campo quirúrgico, resulta más complicada su instalación.

Los efectos colaterales del BSC son similares a los del BIE, aunque menos frecuentes. Por ejemplo, la PHD puede alcanzar un $60 \%$ con inyecciones intra plexo[8] y el síndrome Horner superar el 30\%[2]. Al igual que en el $\mathrm{BIE}$, estos efectos dependen del volumen de $\mathrm{AL}$ y el sitio de inyección. Por último, especial consideración se debe tener con la identificación de estructuras vasculares y pleura, y su relación con el

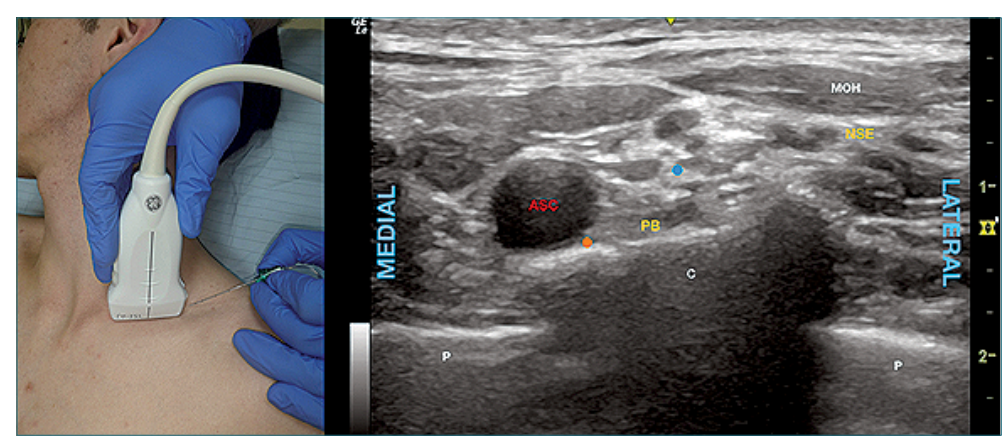

Figura 4. A. Posición transductor BSC; B. Sonoanatomía BSC: $\mathrm{MOH}$ : músculo omohioideo; P: pleura; C: primera costilla; ASC: arteria subclavia; PB: plexo braquial; NSE: nervio supraescapular. Punto azul: sitio de inyección intracluster; Punto naranjo: punto de inyección "corner pocket". 
trayecto y la punta de la aguja.

\section{Bloqueo infraclavicular}

El bloqueo infraclavicular (BIC) aborda el plexo a nivel de los cordones, representando una alternativa de altamente eficaz de bloqueo anestésico y analgésico desde el codo hacia distal. No obstante, dada la profundidad variable del objetivo de inyección que determina un ángulo de punción pronunciado que puede perjudicar la óptima visualización de la aguja, tiende a ser poco popular[26].

Las divisiones del PB en su paso entre la clavícula y la primera costilla conforman los cordones del plexo, manteniéndose estos inicialmente aglomerados lateralmente a la arteria subclavia/axilar[5] para luego distribuirse alrededor de la AA a nivel de la apófisis coracoides.

A nivel costoclavicular el $\mathrm{CL}$, a pesar de estar muy cerca del medial y posterior, pudiera encontrarse en un compartimento conectivo distinto[5],[27].

Además, a pesar de la nomenclatura, estudios de resonancia nuclear, han mostrado que, aunque en el mismo plano aponeurótico, la ubicación final de los cordones alrededor de la AA es bastante variable siendo lo más frecuente que adopten una ubicación pósterolateral a la arteria[6].

A pesar de que el BIC puede ser ejecutado con $\mathrm{NE}$, la adición de guía ultrasonográfica ha demostrado acortar los tiempos de procedimiento y la latencia, y a su vez ha prolongado la duración del efecto. Más aún, el US incluso sería superior a US más NE, acortando los tiempos y aumentado el éxito del procedimiento[2].

En el abordaje clásico del BIC, con el paciente en posición supina y un grado variable de abducción del hombro se ubica el transductor en un plano parasagital medial a la apófisis coracoides (Figura 5A) hasta identificar la imagen en eje corto de la AA profunda a los músculos pectorales mayor y menor (Figura 5B). La punción se realiza en plano con el US insertando la aguja desde craneal a caudal entre clavícula y trans- ductor.

En el abordaje retroclavicular, con menos evidencia a su haber, para mejorar la visión ultrasonográfica de la aguja, con el mismo posicionamiento del transductor e imagen del BIC clásico, la aguja se inserta por detrás de la clavícula en dirección caudal, quedando la primera porción del avance de ésta oculta tras la sombra acústica clavicular, pero con una mejor visualización de la aguja al acercarse al objetivo por estar más perpendicular al US[28]. Sin embargo, a pesar de que debiera reflejarse en una menor dificultad con el procedimiento, la evidencia en términos de tiempos de procedimiento es ambigua, y ciertas consideraciones deben tenerse por el riesgo de dañar estructuras frecuentemente ubicadas en el trayecto retroclavicular de la aguja, específicamente el nervio y vena supraescapular[29],[26].

Alternativamente, en el abordaje costoclavicular descrito recientemente[5], posicionando el transductor bajo la clavícula, pero paralelo a ésta y con inclinación craneal (Figura 6A) es posible identificar los tres cordones del plexo bajo el músculo subclavio y lateral a la arteria subclavia/axilar, (Figura 6B). Así, en plano con el US se introduce la aguja de lateral a medial. Evidencia creciente con este abordaje sugiere que manteniendo los volúmenes de inyección controlados es posible incluso dar analgesia equivalente a BIE en cirugía de hombro sin riesgo de PHD[30].

Existen dosis (VEM90\% de ALs), determinadas por metodologías validadas para anestesia quirúrgica de ES para los abordajes clásico y costoclavicular, siendo $35 \mathrm{~mL}$ y $34 \mathrm{~mL}$ respectivamente[31],[32]. Para el abordaje retroclavicular, con una metodología menos precisa, el VEM95\% oscilaría entre $18 \mathrm{~mL}$ y 37 $\mathrm{mL}[33]$.

Respecto al sitio ideal de inyección bastaría con una inyección dorsal a la arteria para inyecciones a nivel paracoracoideo independiente del abordaje, pero utilizando un volumen adecuado. Es importante obtener el signo de doble burbuja inicialmente generado por el $\mathrm{AL}$ inyectado bajo al $\mathrm{AA}$, y luego confir-

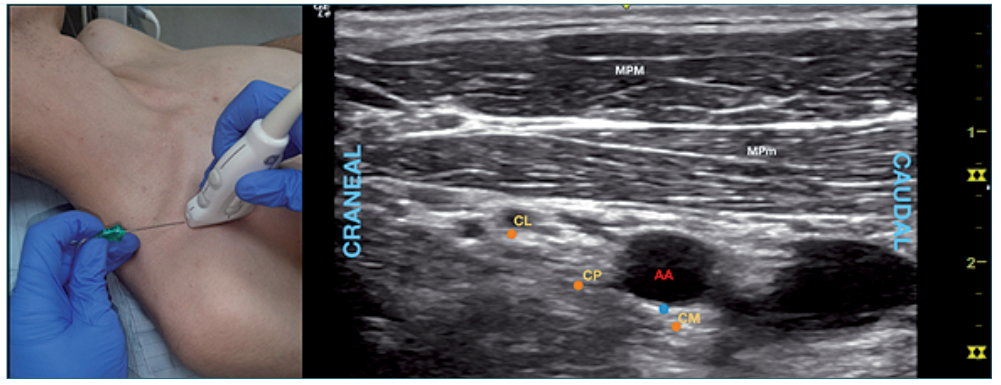

Figura 5. A. Posición transductor BIC; B. Sonoanatomía BIC: MPM: músculo pectoral mayor; MPm: músculo pectoral menor; AA: arteria axilar; CL: cordón lateral; CP: cordón posterior; CM: cordón medial. Punto azul: sitio de inyección periarterial; Puntos naranjos: puntos de inyección perineurales. 


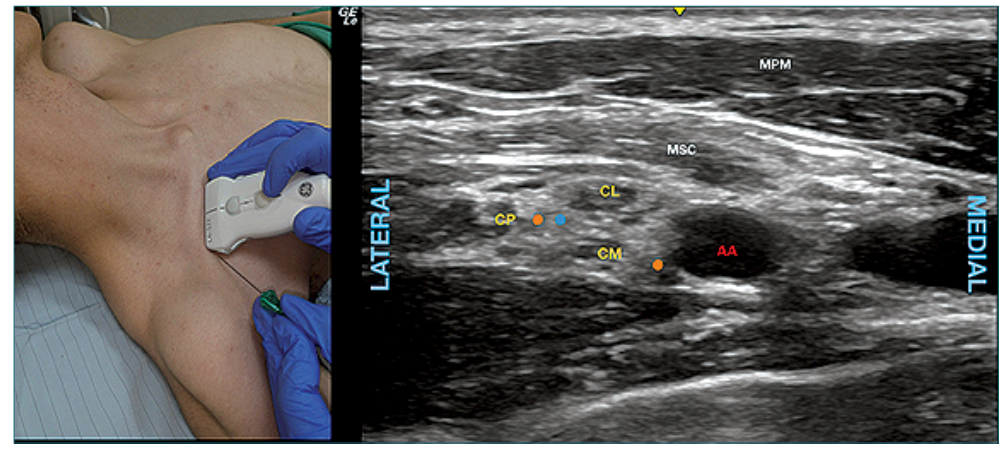

Figura 6. A. Posición transductor $\mathrm{BCC}$; B. Sonoanatomía BCC: MPM: músculo pectoral mayor; MSC: músculo subclavio; AA: arteria axilar; CL: cordón lateral; CP: cordón posterior; CM: cordón medial. Punto azul: sitio de inyección entre cordones; Puntos naranjos: alternativas de inyección asumiendo compartimento conectivo.

mar como ésta es comprimida y desplazada, e incluso acústicamente borrada por el anestésico que la rodea[34]. Aún está pendiente resolver si en el abordaje costoclavicular pudiera ser mejor realizar inyecciones múltiples por sobre lo descrito hasta ahora con una sola inyección entre los tres cordones[5],[27],[35].

En general el BIC representa una buena alternativa para la instalación de catéteres, pero al igual que en el BSC se debe contemplar para las dosis de bolo e infusión la dispersión anatómica en que pueden encontrarse los cordones según el abordaje.

Los abordajes infraclaviculares del PB no están exentos de algunos de los efectos colaterales encontrados en los abordajes por sobre la clavícula. La incidencia de estos sería volumen dependiente y así por ejemplo es que tanto con la versión clásica como costoclavicular utilizando sus VEM90 se describe un $8 \%$ de $\mathrm{PHD}[35]$ que, sin embargo, se reduce a $0 \%$ utilizando $20 \mathrm{~mL}[30],[36]$.

Las inyecciones únicas peri arteriales pudieran tener distinta eficacia ante la presencia de variaciones anatómicas como arterias duplicadas o luego de cirugías en la región infraclavicular. Por último, la presencia de marcapasos o reservorios de quimioterapia revisten contraindicaciones propias de estos abordajes.

\section{Bloqueo axilar}

El bloqueo axilar (BA) es indicado principalmente para cirugía desde antebrazo a distal con buen perfil de eficacia y seguridad en su versión guiada por US.

A nivel medioaxilar ya es posible encontrar los principales nervios terminales que inervan la extremidad superior distal al hombro distribuidos alrededor de la arteria axilar siendo el primero que abandona esta ubicación el NMC y que generalmente es ubicable en un trayecto interfascial o intramuscular entre el coracobraquial y el bíceps braquial[1]. Luego en el primer tercio humeral se desprenderá el NR hacia su trayecto posterior rodeando el tercio medio del húmero hasta ubicarse en una posición lateral a nivel del codo. Subsecuentemente, ocurrirá algo similar con el UN, que a nivel del codo cruza por el canal cubital y, por último, sólo el NM permanece en todo su trayecto hasta el codo en una íntima relación con la arteria.

Respecto a la neurolocalización, tanto por reparos, con inyecciones peri o transarteriales y como con NE se logra adecuada eficacia, sin embargo, la adición de US permite disminuir los tiempos de procedimiento y punciones vasculares, aumenta la tasa de éxito, siendo más eficientes las punciones exclusivamente ecoguiadas[2].

Se puede escanear inicialmente desde la axila a distal tratando de identificar la emergencia de los nervios desde la vaina vascular y localizar el punto ideal de punción. Así, con el hombro abducido en rotación externa y la axila expuesta se posiciona el transductor a este nivel (Figura 7A) para obtener una imagen en eje corto de la AA (Figura 7B). Con una técnica en plano desde anterior a posterior se dirige primero la aguja hacia el NMC y luego se redirige hacia la AA para el bloqueo de los nervios restantes.

La evidencia propone que tanto inyecciones múltiples como únicas poseen igual éxito de bloqueo, siendo más eficiente en términos de tiempo de procedimiento y número de pases de aguja emplear una inyección a nivel del NMC y una inyección perivascular en el dorso de la arteria, logrando que la silueta de esta desaparezca al rodearse por AL[37].

La dosis (VEM 90\%) demostrada con metodología adecuada son $6 \mathrm{~mL}$ alrededor del NMC y $24 \mathrm{~mL}$ periarterial[38]. En caso de inyecciones proximales donde no se visualice el musculocutáneo se pueden inyectar los $30 \mathrm{~mL}$ periarterial asumiendo que el NMC aún estaría presente en la vaina perivascular.

La axila es un lugar complejo para la instalación de catéteres dado su alta movilidad y sudoración. De 


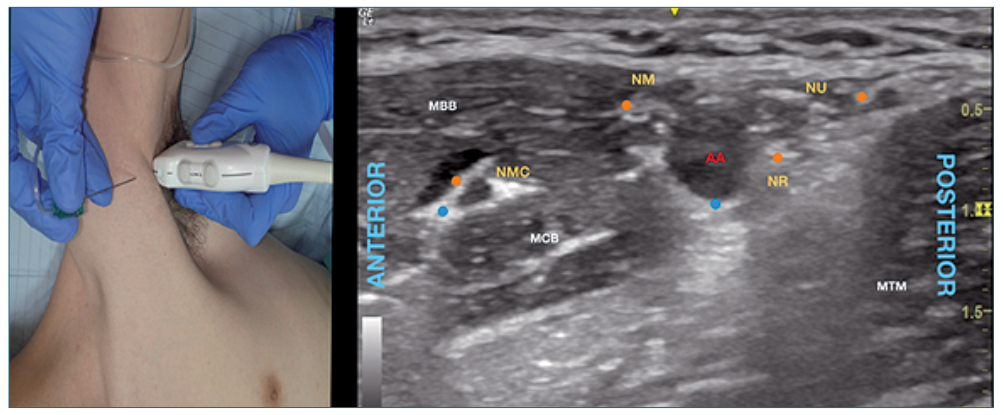

Figura 7. A. Posición transductor BA; B. Sonoanatomía BA: MBB: músculo bíceps braquial; MCB: músculo coracobraquial; MTM: músculo teres menor; $A A$ : arteria axilar; NMC: nervio musculocutáneo; NM: nervio mediano; NR: nervio radial; UN: nervio ulnar. Puntos azules: sitios de inyección para bloqueo de NMC y periarterial para los otros tres nervios; Puntos naranjos: punto de inyección perineurales dirigidos en caso de visualización.

instalarse, se recomienda la ubicación de la punta del catéter dorsal a la arteria[1].

Es muy relevante siempre considerar que rodeando la arteria puede existir un número variable de venas que serán fácilmente colapsadas al posicionar el transductor de ultrasonido y por lo cual se debe tener especial cuidado en evitar o detectar precozmente signos de inyección intravascular. Además, ante la presencia de variaciones anatómicas arteriales (arteria duplicada), la inyección única periarterial puede perder eficacia.

\section{Bloqueos selectivos}

En la búsqueda de obtener analgesia de hombro mediante bloqueos regionales, pero con menor incidencia de bloqueo del nervio frénico se han estudiado alternativas basadas en la lógica de que la PHD se produce porque proximal a la clavícula el PB y el NF están muy cerca. Así, inyectando AL de manera más selectiva y a mayor distancia del trayecto frénico la incidencia de PHD y una eventual complicación respiratoria en pacientes susceptibles debiera ser menor. Por esto, se han estudiado alternativas como el bloqueo de los nervios axilar a nivel del cuello humeral y supraescapular en la escotadura de la escápula y más recientemente, el bloqueo selectivo del tronco superior y el bloqueo anterior del nervio supraescapular.

\section{Bloqueo del tronco superior}

El bloqueo del tronco superior (BTS) se describió en 2014[39], y consiste en ubicar el tronco superior en el momento en que se está dividiendo, pero antes de que el NSE inicie su recorrido hacia la escápula[8]. La lógica tras esta técnica radica en que a nivel interescalénico donde ubicamos habitualmente $\mathrm{C5}-\mathrm{C} 6$, el nervio frénico está a $2 \mathrm{~mm}$ de distancia, sin embargo, esta distancia aumenta progresivamente hacia distal, lo que permitiría que inyecciones a nivel del tronco superior tengan menos incidencia de parálisis frénica si se evita la difusión anterior del AL[7].

Anatómicamente, la inyección de $5 \mathrm{~mL}$ de tinción en cadáveres, lateral al tronco superior, respetaría el NF. A la fecha solo existen dos estudios randomizados comparando el BTS con BIE evaluando PHD. En el primero, un promisorio $5 \%$ de PHD total fue reportado utilizando $15 \mathrm{~mL}$ de $\mathrm{AL}[40]$, no obstante, en un estudio posterior, utilizando la misma técnica y volumen de AL, a pesar de replicarse el 5\% de PHD total, también se reportó un $70 \%$ de PHD parcial, para la cual no está claro el rango de compromiso respiratorio al cual puede estar asociado[41]. Así, estudios clínicos evaluando BTS con dosis menores de AL debieran ejecutarse antes de utilizar esta técnica en pacientes de elevado riesgo respiratorio[8].

\section{Bloqueo posterior y anterior del nervio supraescapular}

Describiéndose el nervio supraescapular (NSE) como el responsable de $70 \%$ de la inervación sensitiva de la articulación del hombro[8] es entendible porque existe abundante evidencia respecto al rol de su bloqueo intentando obtener analgesia de hombro tanto en el contexto de dolor crónico como agudo perioperatorio. Clásicamente se ha bloqueado a través de diversas técnicas de localización a nivel de la escotadura escapular obteniendo resultados variables de analgesia. Sin embargo, en el contexto postoperatorio de hombro, a pesar de demostrar superioridad a placebo o infiltración periarticular con ALs, sería inferior a BIE[8].

En la técnica guiada por US para el abordaje posterior a este bloqueo se posiciona el transductor sobre la espina escapular con cierta orientación caudal (Figura $8 \mathrm{~A}$ ) hasta localizar la sombra acústica generada 

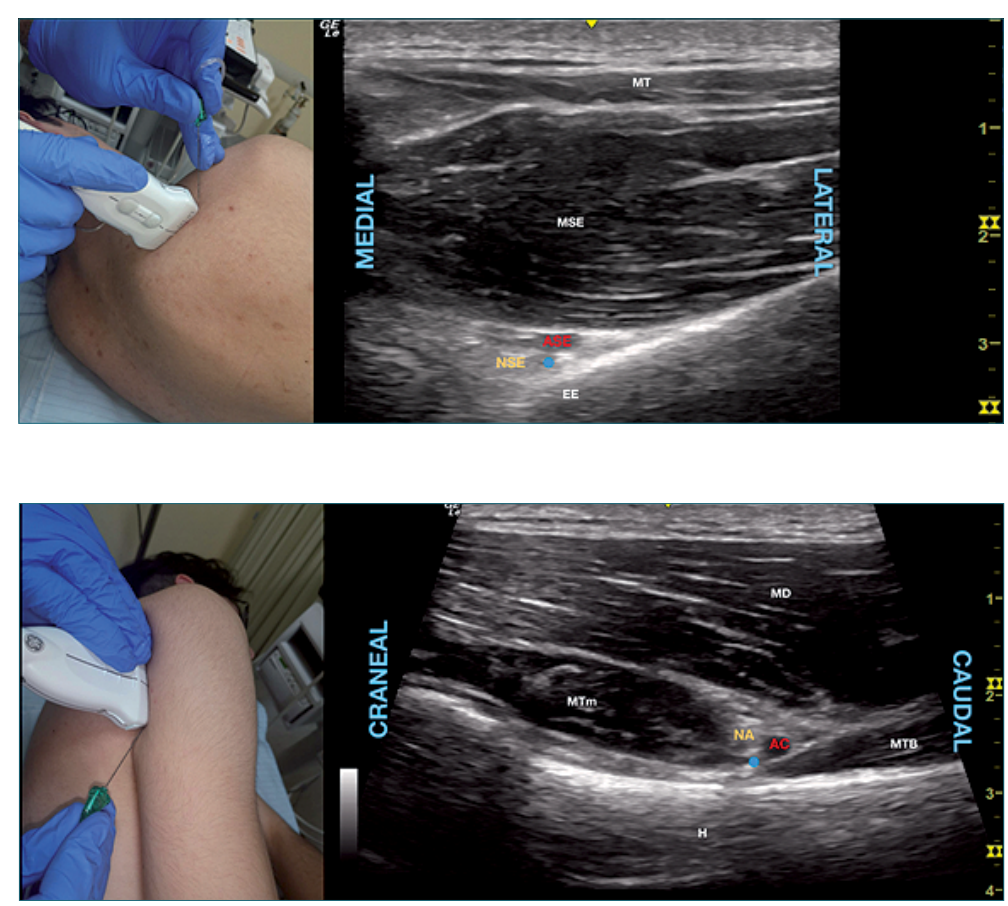

Figura 8. A. Posición transductor BNSEP; $\boldsymbol{B}$. Sonoanatomía BNSEP. MT: músculo trapecio; MSE: músculo supraescapular; ASE: arteria supraescapular; EE: escotadura escapular; NSE: nervio supraescapular. Punto azul: sitio de inyección.

Figura 9. A. Posición transductor BNA; B. Sonoanatomía BNA: MD: músculo deltoides; MTm: músculo teres menor; MTB: músculo tríceps braquial; $H$ : húmero; AC: arteria circunfleja; NA: nervio axilar. Punto azul: sitio de inyección intrafascial. por la espina escapular y luego se ubica la escotadura donde se puede identificar frecuentemente los vasos supraescapulares que mantienen una íntima relación con el nervio (Figura 8B). Independiente de si estas estructuras son o no visualizadas, el objetivo de inyección es el plano entre el músculo supraespinoso y el periostio cercano a la escotadura dirigiendo la aguja con una técnica en plano con el US, ya sea desde medial a lateral o lateral a medial e inyectando entre 5 a $15 \mathrm{~mL}$ de AL buscando identificar como el músculo se separa del periostio con la inyección[36],[42].

Una explicación a la inferioridad del abordaje posterior del NSE es que hay otros nervios involucrados en la inervación del hombro. Sin embargo, en un estudio donde se bloqueó el NSE en la escotadura y además se bloquearon los restantes nervios involucrados mediante un bloqueo de los cordones del PB se mantuvo inferioridad analgésica respecto a BIE en el postoperatorio inmediato[36]. Así, otra explicación complementaria es que en un porcentaje no menor de pacientes el NSE emite un ramo articular antes de entrar en la escotadura y talvez no sea bloqueado en abordajes posteriores[43]. Entonces, una solución lógica sería bloquear el NSE selectivamente por vía anterior, una vez que abandona el tronco superior, para lo cual es relevante su relación con el músculo omohioideo[20]. El problema es que a pesar de que es posible bloquear selectivamente el NSE con este abordaje inyectando volúmenes bajos de AL[44],los ensayos clínicos que han explorado esta técnica han utilizado volúmenes superiores a $10 \mathrm{~mL}$ de $\mathrm{AL}[45],[46]$, sabiéndose desde estudios cadavéricos que puede haber hasta un $20 \%$ de difusión hasta el nervio frénico con este volumen[47] y además, es imposible determinar si la equivalencia analgésica con BIE fue debida finalmente a la inclusión del ramo articular del NSE o porque se está en presencia de un BSC de bajo volumen similar a la técnica con inyección pósterolateral ya descrita[25], dada la cercanía con el racimo principal de divisiones del PB a esta altura (Figura 4B).

\section{Bloqueo de nervio axilar}

Una alternativa simple de complementar el bloqueo del NSE para el manejo del dolor de hombro es agregar un bloqueo del nervio axilar a nivel del cuello humeral. La combinación de estos dos bloqueos es superior analgésicamente al bloqueo del NSE por sí solo, sin embargo, es inferior a BIE en cirugía mayor de hombro, representando una alternativa para analgesia en cirugía con menor carga de dolor[8]. Existen variantes en la técnica descrita, pero en general con el codo flectado y hombro en rotación interna se posiciona un transductor de US a nivel del cuello humeral por posterior paralelo a su eje largo (Figura 
9A) ubicando el espacio cuadrangular determinado por los músculos teres menor y mayor y las cabezas larga y lateral del tríceps braquial, aproximadamente $2 \mathrm{~cm}$ distal al acromion por posterior, identificándose el nervio axilar y los vasos circunflejos profundos al músculo deltoides en el borde distal del teres menor
(Figura 9B)[42],[48]. Ante la duda el uso de doppler color puede ayudar a identificar los vasos y el nervio que habitualmente se encuentra craneal a estos.

Dosis entre 8 a $15 \mathrm{ml}$ de $\mathrm{AL}$ se han utilizado con éxito.

\section{Referencias}

1. Tran D, Neal J. Upper Extremity Blocks. Neal J, Tran DQ, Salinas F. A Practical Approach to Regional Anesthesia and Acute Pain Medicine. Quinta Edición. Wolters-Kluwer Health. Philadelphia. 2018. p-99-117. https://doi.org/ 10.2174/978168108721411801 0005.

2. Choi S, McCartney CJ. Evidence Base for the Use of Ultrasound for Upper Extremity Blocks: 2014 Update. Reg Anesth Pain Med. 2016 Mar-Apr;41(2):242-50. https://doi.org/10.1097/ AAP.0000000000000155 PMID:25376973

3. Tran DQ, Dugani S, Asenjo JF. Upper extremity Nerve Blocks. In: Kaye A, Urman R, Vadivelu $N$, editors. Essentials of regional anesthesia. New York: Springer; 2012. https://doi. org/10.1007/978-1-4614-10133_13.

4. Tran DQ, Tiyaprasertkul W, González AP. Analgesia for clavicular fracture and surgery: a call for evidence. Reg Anesth Pain Med. 2013 Nov-Dec;38(6):539-43. https://doi.org/10.1097/ AAP.0000000000000012 PMID:24121609

5. Sala-Blanch $X$, Reina MA, Pangthipampai $\mathrm{P}$, Karmakar MK. Anatomic Basis for Brachial Plexus Block at the Costoclavicular Space: A Cadaver Anatomic Study. Reg Anesth Pain Med. 2016 May-Jun;41(3):387-91. https://doi.org/10.1097/ AAP.0000000000000393
PMID:27035461

6. Sauter AR, Smith HJ, Stubhaug A, Dodgson MS, Klaastad $\varnothing$. Use of magnetic resonance imaging to define the anatomical location closest to all three cords of the infraclavicular brachial plexus. Anesth Analg. 2006 Dec;103(6):1574-6. https://doi.org/10.1213/01. ane.0000242529.96675.fd PMID:17122242

7. Tran DQ, Elgueta MF, Aliste J, Finlayson RJ. DiaphragmSparing nerve blocks for shoulder surgery. Reg Anesth Pain Med. 2017 Jan/Feb;42(1):328. https://doi.org/10.1097/ AAP.0000000000000529 PMID:27941477

8. Tran DQ, Layera S, Bravo D, Cristi-Sánchez I, Bermúdez L,Aliste J. Diaphragm-sparing nerve blocks for shoulder surgery, revisited. Reg Anesth Pain Med. Epub ahead of print. https://doi.org/10.1136/rapm2019-100908.

9. Spence BC, Beach ML, Gallagher JD, Sites BD. Ultrasoundguided interscalene blocks: understanding where to inject the local anaesthetic. Anaesthesia. 2011 Jun;66(6):509-14. https://doi.org/10.1111/ j.1365-2044.2011.06712.x PMID:21568985

10. Franco $C D$, Williams JM, Williams JM. Ultrasound-Guided Interscalene Block: Reevaluation of the "Stoplight" Sign and Clinical Implications. Reg Anesth Pain Med. 2016 Jul-Aug;41(4):452-9. https://doi.org/10.1097/

\section{AAP. 0000000000000407 PMID:27203394}

11. Hanson NA, Auyong DB. Systematic ultrasound identification of the dorsal scapular and long thoracic nerves during interscalene block. Reg Anesth Pain Med. 2013 Jan-Feb;38(1):54-7. https://doi.org/10.1097/ AAP.0b013e31826f0a63 PMID:23080352

12. Mariano ER, Loland VJ, Ilfeld BM. Interscalene perineural catheter placement using an ultrasound-guided posterior approach. Reg Anesth Pain Med. 2009 Jan-Feb;34(1):60-3. https://doi.org/10.1097/ AAP.0b013e3181933af7 PMID:19258989

13. Fredrickson MJ, Ball CM, Dalgleish AJ. Posterior versus anterolateral approach interscalene catheter placement: a prospective randomized trial. Reg Anesth Pain Med. 2011 Mar-Apr;36(2):125-33. https://doi.org/10.1097/ AAP.0b013e31820d5ee6 PMID:21425511

14. Falcão LF, Pérez MV, de Castro I, Yamashita AM, Tardelli MA, Amaral JL. Minimum effective volume of $0.5 \%$ bupivacaine with epinephrine in ultrasoundguided interscalene brachial plexus block. Br J Anaesth. 2013 Mar;110(3):450-5. https:// doi.org/10.1093/bja/aes419 PMID:23195326

15. Gautier P, Vandepitte $C$, Ramquet $C$, DeCoopman M, Xu $D$, Hadzic A. The minimum effective anesthetic volu- 
me of $0.75 \%$ ropivacaine in ultrasound-guided interscalene brachial plexus block. Anesth Analg. 2011 Oct;113(4):9515. https://doi.org/10.1213/ ANE.0b013e31822b876f PMID:21821517

16. Fredrickson MJ, Abeysekera A, White R. Randomized study of the effect of local anesthetic volume and concentration on the duration of peripheral nerve blockade. Reg Anesth Pain Med 2012 Sep-Oct;37(5):495-501. https://doi.org/10.1097/ AAP.0b013e3182580fdo PMID:22664978

17. Palhais N, Brull R, Kern C, Jacot-Guillarmod A, Charmoy A, Farron A, et al. Extrafascial injection for interscalene brachial plexus block reduces respiratory complications compared with a conventional intrafascial injection: a randomized, controlled, double-blind trial. Br J Anaesth. 2016 Apr;116(4):531-7. https:// doi.org/10.1093/bja/aew028 PMID:26994230

18. Albrecht E, Bathory I, Fournier N, Jacot-Guillarmod A, Farron A, Brull R. Reduced hemidiaphragmatic paresis with extrafascial compared with conventional intrafascial tip placement for continuous interscalene brachial plexus block: a randomized, controlled, double-blind trial. $\mathrm{Br}$ J Anaesth. 2017 Apr;118(4):58692. https://doi.org/10.1093/bja/ aex050 PMID:28403412

19. El-Boghdadly K, Chin KJ, Chan VW. Phrenic Nerve Palsy and Regional Anesthesia for Shoulder Surgery: Anatomical, Physiologic, and Clinical Considerations. Anesthesiology. 2017 Jul;127(1):17391. https://doi.org/10.1097/ ALN.0000000000001668 PMID:28514241

20. Siegenthaler A, Moriggl B, Mlekusch S, Schliessbach J,
Haug M, Curatolo M, et al. Ultrasound-guided suprascapular nerve block, description of a novel supraclavicular approach. Reg Anesth Pain Med. 2012 May-Jun;37(3):325-8.

https://doi.org/10.1097/

AAP.0b013e3182409168 PMID:22222688

21. Kant A, Gupta PK, Zohar S, Chevret S, Hopkins PM. Application of the continual reassessment method to dose-finding studies in regional anesthesia: an estimate of the ED95 dose for 0.5\% bupivacaine for ultrasound-guided supraclavicular block. Anesthesiology. 2013 Jul;119(1):2935. https://doi.org/10.1097/ ALN.0b013e31829764cf PMID:23648519

22. Tran DQ, Dugani $S$, Correa JA, Dyachenko A, Alsenosy N, Finlayson RJ. Minimum effective volume of lidocaine for ultrasound-guided supraclavicular block. Reg Anesth Pain Med. 2011 Sep-Oct;36(5):466-9. https://doi.org/10.1097/ AAP.0b013e3182289f59 PMID:21857275

23. Techasuk W, González AP, Bernucci F, Cupido T, Finlayson RJ, Tran DQ. A randomized comparison between double-injection and targeted intracluster-injection ultrasound-guided supraclavicular brachial plexus block. Anesth Analg. 2014 Jun;118(6):13639. https://doi.org/10.1213/ ANE.0000000000000224 PMID:24842181

24. Tran DQ, Muñoz L, Zaouter C, Russo G, Finlayson RJ. A prospective, randomized comparison between single- and doubleinjection, ultrasound-guided supraclavicular brachial plexus block. Reg Anesth Pain Med. 2009 Sep-Oct;34(5):420-4. https://doi.org/10.1097/
AAP.0b013e3181ae733a

PMID:19920418

25. Aliste J, Bravo D, Fernández D, Layera S, Finlayson RJ, Tran DQ. A Randomized Comparison Between Interscalene and Small-Volume Supraclavicular Blocks for Arthroscopic Shoulder Surgery. Reg Anesth Pain Med. 2018 Aug;43(6):5905. https://doi.org/10.1097/ AAP. 0000000000000767 PMID:29630033

26. Leurcharusmee $P$, Layera $S$, Finlayson RJ, Tran DQ. Retroclavicular approach to infraclavicular brachial plexus block: a logical conundrum. Reg Anesth Pain Med. 2019 Jan;44(2):273. https://doi.org/10.1136/rapm2018-100133 PMID:30635512

27. Monzó E, Hadzic A. Costoclavicular approach to the brachial plexus block: simple or double injection? Reg Anesth Pain Med. 2019 Sep;rapm-2019-100852.; Epub ahead of print. https://doi. org/10.1136/rapm-2019-100852 PMID:31570494

28. Charbonneau J, Fréchette $Y$, Sansoucy $Y$, Echave P. The UItrasound-Guided Retroclavicular Block: A Prospective Feasibility Study. Reg Anesth Pain Med. 2015 Sep-Oct;40(5):605-9. https://doi.org/10.1097/ AAP. 0000000000000284 PMID:26222346

29. Sancheti SF, Uppal V, Sandeski R, Kwofie MK, Szerb JJ. A Cadaver Study Investigating Structures Encountered by the Needle During a Retroclavicular Approach to Infraclavicular Brachial Plexus Block. Reg Anesth Pain Med. 2018 Oct;43(7):7525. https://doi.org/10.1097/ AAP. 0000000000000826 PMID:29923952

30. Aliste J, Bravo D, Layera S, Fernández D, Jara Á, Maccioni C, et al. Randomized comparison between interscalene and costo- 
clavicular blocks for arthroscopic shoulder surgery. Reg Anesth Pain Med. 2019 Jan;44(4):472-7. https://doi.org/10.1136/rapm2018-100055 PMID:30635497

31. Tran DQ, Dugani S, Dyachenko A, Correa JA, Finlayson RJ. Minimum effective volume of lidocaine for ultrasound-guided infraclavicular block. Reg Anesth Pain Med. 2011 Mar-Apr;36(2):190-4. https://doi.org/10.1097/ AAP.0b013e31820d4266 PMID:21270721

32. Sotthisopha T, Elgueta MF, Samerchua A, Leurcharusmee P, Tiyaprasertkul W, Gordon A, Finlayson RJ, Tran DQ. Minimum Effective Volume of Lidocaine for Ultrasound-Guided Costoclavicular Block. Reg Anesth Pain Med 2017;42: 571.574. https://doi.org/10.1097/ AAP.0000000000000629.

33. Kavakli AS, Kavrut Ozturk N, Arslan U. Volume mínimo efetivo de bupivacaína a 0,5\% para abordagem retroclavicular guiada por ultrassom no bloqueio do plexo braquial por via infraclavicular. Rev Bras Anestesiol. 2019 May - Jun;69(3):253-8. https://doi. org/10.1016/j.bjan.2018.11.011 PMID:31030903

34. Tran DQ, Bertini $P$, Zaouter C, Muñoz L, Finlayson RJ. A prospective, randomized comparison between single- and double-injection ultrasoundguided infraclavicular brachial plexus block. Reg Anesth Pain Med. 2010 Jan-Feb;35(1):16-21. https://doi.org/10.1097/ AAP.0b013e3181c7717c PMID:20048654

35. Songthamwat B, Karmakar MK, Li JW, Samy W, Mok LY. Ultrasound-Guided Infraclavicular Brachial Plexus Block: Prospective Randomized Comparison of the Lateral Sagittal and Costoclavicular Approach. Reg Anesth Pain Med. 2018 Nov;43(8):825-
31. https://doi.org/10.1097/ AAP.0000000000000822 PMID:29923950

36. Aliste J, Bravo D, Finlayson RJ, Tran DQ. A randomized comparison between interscalene and combined infraclavicular-suprascapular blocks for arthroscopic shoulder surgery. Can J Anaesth. 2018 Mar;65(3):280-7. https:// doi.org/10.1007/s12630-0171048-0 PMID:29270914

37. Tran DQ, Pham K, Dugani S, Finlayson RJ. A prospective, randomized comparison between double-, triple-, and quadruple-injection ultrasoundguided axillary brachial plexus block. Reg Anesth Pain Med. 2012 May-Jun;37(3):248-53. https://doi.org/10.1097/ AAP.0b013e31824611bf PMID:22354104

38. González AP, Bernucci F, Pham K, Correa JA, Finlayson RJ, Tran DQ. Minimum effective volume of lidocaine for doubleinjection ultrasound-guided axillary block. Reg Anesth Pain Med. 2013 Jan-Feb;38(1):16-20. https://doi.org/10.1097/ AAP.0b013e3182707176 PMID:23146999

39. Burckett-St Laurent D, Chan V, Chin KJ. Refining the ultrasoundguided interscalene brachial plexus block: the superior trunk approach. Can J Anaesth. 2014 Dec;61(12):1098-102. https:// doi.org/10.1007/s12630-0140237-3 PMID:25208976

40. $\operatorname{Kim} D H$, Lin $Y$, Beathe JC, Liu J, Oxendine JA, Haskins SC, et al. Superior trunk block: a phrenic-sparing alternative to the interscalene block: a randomized controlled trial. Anesthesiology. 2019 Sep;131(3):52133. https://doi.org/10.1097/ ALN.0000000000002841 PMID:31283740

41. Kang R, Jeong JS, Chin KJ, Yoo JC, Lee JH, Choi SJ, et al.
Superior Trunk Block Provides Noninferior Analgesia Compared with Interscalene Brachial Plexus Block in Arthroscopic Shoulder Surgery. Anesthesiology. 2019 Dec;131(6):131626.; epub ahead of print. https://doi.org/10.1097/ ALN.0000000000002919 PMID:31490292

42. Dhir S, Sondekoppam RV, Sharma R, Ganapathy S, Athwal GS. A Comparison of Combined Suprascapular and Axillary Nerve Blocks to Interscalene Nerve Block for Analgesia in Arthroscopic Shoulder Surgery: An Equivalence Study. Reg Anesth Pain Med. 2016 Sep-Oct;41(5):564-71. https://doi.org/10.1097/ AAP.0000000000000436 PMID:27380105

43. Vorster W, Lange CP, Briët RJ, Labuschagne BC, du Toit DF, Muller CJ, et al. The sensory branch distribution of the suprascapular nerve: an anatomic study. J Shoulder Elbow Surg. 2008 May-Jun;17(3):500-2. https://doi. org/10.1016/j.jse.2007.10.008 PMID:18262803

44. Rothe $C$, Steen-Hansen $C$, Lund J, Jenstrup MT, Lange KH. Ultrasound-guided block of the suprascapular nerve - a volunteer study of a new proximal approach. Acta Anaesthesiol Scand. 2014 Nov;58(10):122832. https://doi.org/10.1111/ aas.12392 PMID:25186626

45. Auyong DB, Hanson NA, Joseph RS, Schmidt BE, Slee AE, Yuan SC. Comparison of Anterior Suprascapular, Supraclavicular, and Interscalene Nerve Block Approaches for Major Outpatient Arthroscopic Shoulder Surgery: A Randomized, Double-blind, Noninferiority Trial. Anesthesiology. 2018 Jul;129(1):47-57. https://doi.org/10.1097/ ALN.0000000000002208 
PMID:29634491

46. Wiegel M. moriggi B, Scwarkzopf $P$, Petroff D, Reske AW. Anterior Sprascapular Nerve Block Versus Interscalene Brachial Plexus Block for Shoulder in the Outpatient Setting: A randomized Controlled Patient- and Assessor -Blinded Trial. Reg Anesth Pain Med. 2017;42(3):310-8. https://doi.org/10.1097/

AAP.0000000000000573

PMID:28257388

47. Laumonerie $P$, Ferré $F$, Cances J, Tibbo ME, Roumiguié $M$, Mansat $P$, et al. Ultrasound-guided proximal suprascapular nerve block: A cadaveric study. Clin Anat. 2018 Sep;31(6):824-9. https:// doi.org/10.1002/ca.23199
PMID:29732608

48. Rothe C, Asghar S, Andersen HL, Christensen JK, Lange KH. Ultrasound-guided block of the axillary nerve: a volunteer study of a new method. Acta Anaesthesiol Scand. 2011 May;55(5):56570. https://doi.org/10.1111/ j.1399-6576.2011.02420.x PMID:21827442 